\title{
ARTICLE
}

\section{Dose dependence and uncertainty evaluation of calibration factors for active neutron personal dosimeters}

\author{
Jungho Kim*, Hyeonseo Park, Kil-Oung Choi and Jiyeon Kwak \\ Korea Research Institute of Standards and Science, Daejeon, 305-340, Rep. of Korea
}

\begin{abstract}
Active neutron personal dosimeters are now widely used to monitor the neutron personal dose equivalent. They are calibrated periodically in the standard neutron fields such as ${ }^{252} \mathrm{Cf}, \mathrm{D}_{2} \mathrm{O}$-moderated ${ }^{252} \mathrm{Cf}$, and ${ }^{241} \mathrm{Am}$-Be sources. Type B uncertainty of calibration factors should be determined if they are not offered by the manufacturer. DMC $2000 \mathrm{GN}$ and EPD-N2 dosimeters were chosen to evaluate Type B uncertainties. Dose dependence of calibration factors and minimum dose to the dose indication were also investigated with ${ }^{252} \mathrm{Cf}$ source neutrons in the neutron irradiation room at Korea Research Institute of Standards and Science. The neutron dose was irradiated up to around $10 \mathrm{mSv}$ for studying dose dependence. The calibration factor of EPD-N2 was stable above neutron dose around $2 \mathrm{mSv}$ and that of DMC $2000 \mathrm{GN}$ was stable above around 1 $\mathrm{mSv}$. Type B uncertainties of DMC $2000 \mathrm{GN}$ and EPD-N2 were evaluated to be $6 \%$ and $12 \%$ from the serial measurements. Type B uncertainty of EPD-N2 was also evaluated by fitting the normalized normal distribution of calibration factors with Gaussian function from 153 dosimeters. The result was in good agreement with the result from the serial measurement. Study of minimum dose to the dose indication showed that DMC $2000 \mathrm{GN}$ is more sensitive than EPD-N2.
\end{abstract}

Keywords: active neutron personal dosimeter; calibration factor; Type B uncertainty; dose dependence; minimum dose to the dose indication

\section{Introduction}

Neutron personal dosimeters are the devices to measure the neutron personal dose equivalent. They are put on human body and are mounted on the ISO water slab phantom for calibration. The recommended ISO standard neutron fields are the fields produced from the ${ }^{252} \mathrm{Cf},{ }^{241} \mathrm{Am}-\mathrm{Be}$, and $\mathrm{D}_{2} \mathrm{O}$-moderated ${ }^{252} \mathrm{Cf}$ sources. The conventional true value of the neutron personal dose equivalent is calculated by neutron emission rate of a neutron source and fluence to personal dose equivalent conversion coefficients. The conversion coefficients were according to the specifications of ICRP 74 [1] for parallel neutron radiation. In case of real calibration situation, the conversion coefficients of realistically simulated neutron irradiation room could be used instead of ICRP values [2]. Because the conventional true value of the neutron personal ambient dose equivalent is too long, it will be written as in short form of "neutron dose". Calibration factors are determined by readings of the devices and neutron doses.

Nowadays, many active neutron personal dosimeters were developed and are widely used commercially. Many active neutron dosimeters are electronic neutron personal dosimeters and old pocket dosimeters are

*Corresponding author. Email: jungho@kriss.re.kr getting replaced by electronic neutron personal dosimeters. Personal dosimeters are portable devices which are small in size and light in weight. Some dosimeters have small neutron sensors to meet such requirements and neutron sensitivity of personal dosimeters are poor in general compared to neutron ambient dose equivalent meter (neutron surveymeter).

It should be considered that personal dosimeters are usually used in the low dose environment level less than $1 \mathrm{mSv}$. Thus, it is recommended to calibrate the neutron personal dosimeter with the same neutron dose level if the sensitivity of the dosimeter is high enough to evaluate the calibration factor. When calibrations are performed with dosimeters that have low neutron sensitivities, several difficulties exist for evaluating the calibration factor. The most serious problem is the dose dependence of the calibration factor for low dose range. Calibration should not be performed in the neutron dose area where reading is not linear to the reference dose. This limit is also applied to the uncertainty of reading of the dosimeter when it is used in the workplace field.

Another thing to consider for the calibration of personal dosimeters is that personal dosimeters are calibrated only once practically in many cases for saving time and cost. When the calibration is carried out once, the effect of Type A uncertainty (the uncertainty by a statistical analysis of measured quantity values) is minor 
and Type B uncertainty (the uncertainty by means other than a Type A uncertainty) plays a major role. Type A uncertainty derived from repeated measurements for a certain model of instruments could be often transformed into one of the Type B uncertainty for the same instrument model in another measurements. If Type B uncertainty of the dosimeter is not supplied by the manufacturer, it should be evaluated by the person who calibrates the device. Even in case the uncertainty is given by the manufacturer, the calibration field should be carefully considered to apply the value directly.

Two personal neutron dosimeters were chosen for evaluating Type B uncertainty. One is DMC 2000 GN and the other is EPD-N2. EPD-N2 dosimeters are widely used in Korea and are calibrated periodically in the neutron irradiation room with ${ }^{252} \mathrm{Cf}$ source at Korea Research Institute of Standards and Science (KRISS). In this paper, the dose dependence of calibration factors of DMC 2000 GN and EPD-N2 dosimeters were studied and Type B uncertainties were reported.

\section{Experiment}

Thermo Scientific EPD-N2 and MGP DMC 2000GN dosimeters were mounted on the ISO water slab phantom and irradiated with ${ }^{252} \mathrm{Cf}$ neutron source in the neutron irradiation room at KRISS. The dimension of the neutron irradiation room is $6.6 \times 7.6 \times 6.3 \mathrm{~m}^{3}$. The source was installed $\sim 1.5 \mathrm{~m}$ underground and positioned at the center of the room on the remote system [3]. The distance between the source and the phantom surface was $50 \mathrm{~cm}$. A CCTV system was installed to read the values of the device. When the source was on, every change of the reading and the time interval between readings were monitored and recorded. The monitoring screen of the calibration process was recorded by a camcorder until the end of the measurement. The reference of the neutron personal dose equivalent was evaluated from the emission rate of the ${ }^{252} \mathrm{Cf}$ and the measured time. Then, calibration factors were evaluated every change of the reading. These measurements were done with a EPD-N2 and a DMC 2000 GN.

Characteristics of the personal neutron dosimeters were studied: dose dependences of a calibration factor and minimum dose to the dose indication. The reference dose was irradiated up to $10 \mathrm{mSv}$. Every change of reading and time were recorded by using a camcorder. Neutron reference doses were calculated by the irradiation time and the neutron source emission rate which determines the neutron fluence rate at the measurement point.

Type B uncertainties of the DMC $2000 \mathrm{GN}$ and the EPD-N2 were evaluated from serial measurements. Large numbers of calibrations were performed to analyze the data statistically enough. The single irradiation time was 30 minutes and Reference dose was $757.9 \mu \mathrm{Sv}$ for the EPD-N2 and $746.4 \mu \mathrm{Sv}$ for the DMC 2000 GN. Type B uncertainty was extracted from the standard deviation.
Finally, the collection of the calibration factors of EPD-N2 dosimeters was analyzed to evaluate Type B uncertainty. Many EPD-N2 dosimeters are used at nuclear power plant in Korea. More than 1,160 EPD-N2 dosimeters are calibrated periodically every 6 month in the neutron irradiation room with ${ }^{252} \mathrm{Cf}$ source at KRISS. Among them, EPD-N2 dosimeters calibrated more than 5 times were selected for the study of the calibration factor stability. The calibration factors were divided by its average to make normal distribution of calibration factors. Total 153 dosimeters were selected and the number of calibration factors was 1,208. Type B uncertainty of EPD-N2 dosimeter was obtained by Gaussian fitting of the distribution and compared to the previous result.

\section{Results and discussion}

\subsection{Dose dependence of EPD-N2 and DMC 2000 GN}

Figure 1 shows the dose dependence of the calibration factors for the EPD-N2 (black) and the DMC $2000 \mathrm{GN}$ (red). Calibration factors of the EPD-N2 were over 6 below $20 \mu \mathrm{Sv}$ and were omitted in the figure. As dose increases, calibration factors are getting stable and become flat. As shown in the figure, calibration factors of the EPD-N2 fluctuated between $100 \mu \mathrm{Sv}$ and around $2 \mathrm{mSv}$ and were getting stable above $2 \mathrm{mSv}$. For the DMC $2000 \mathrm{GN}$, calibration factors were getting stable above around $1 \mathrm{mSv}$. The final calibration factor for the DMC $2000 \mathrm{GN}$ was 1.48 with $7.833 \mathrm{mSv}$ reference dose and that for the EPD-N2 was 1.53 with $11.363 \mathrm{mSv}$ reference dose. These calibration factors will be compared to those of the serial measurements in section 3.3.

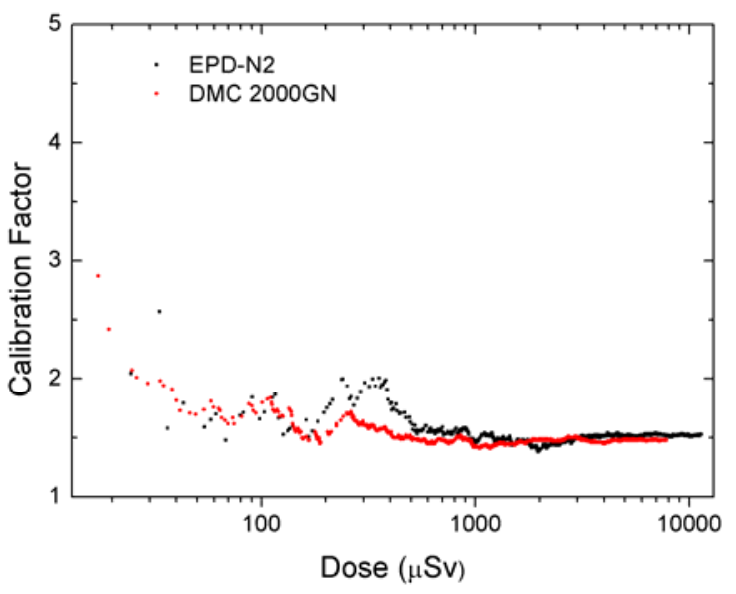

Figure 1. Dose dependence of calibration factors for the EPD-N2 and the DMC 2000 GN dosimeters.

\subsection{Minimum dose to the dose indication}

In this section, we measured a minimum dose required to change a minimum digit of the dose indication. When readings of the dosimeters were changed, readings and the corresponding irradiation times were recorded by using a camcorder. Every 
change of the readings ( $\Delta$ reading) of two dosimeters were compared to the reference doses ( $\Delta$ dose) calculated from time changes. High sensitivity neutron personal dosimeter should respond quickly to neutrons and increase at a fixed rate. This feature is also required to the good linearity of the dosimeter.

The minimum dose to the dose indication for the EPD-N2 and the DMC 2000 GN dosimeters are shown in Figure 2. The horizontal axis is the change of reading ( $\Delta$ reading) and the vertical axis is that of reference dose ( $\Delta$ dose). The top contour plot of Figure 2 is the result of the DMC $2000 \mathrm{GN}$ and shows that reading of the DMC $2000 \mathrm{GN}$ varies by $1 \mu \mathrm{Sv}$ step. The average of reading is $2.05 \mu \mathrm{Sv}$ and the average of dose is $2.76 \mu \mathrm{Sv}$.

The contour plot of the reference dose and the reading for the EPD-N2 is shown in the bottom of Figure 2. Reading values of the EPD-N2 dosimeter are grouped in $1 \mu \mathrm{Sv}, 10 \mu \mathrm{Sv}, 20 \mu \mathrm{Sv}$, and $30 \mu \mathrm{Sv}$. Minimum reading is $1 \mu \mathrm{Sv}$ but most readings are $1 \mu \mathrm{Sv}$ or $10 \mu \mathrm{Sv}$. The average of reading is $4.87 \mu \mathrm{Sv}$ which is the half of $1 \mu \mathrm{Sv}$ and $10 \mu \mathrm{Sv}$ and the average of dose is $7.33 \mu \mathrm{Sv}$. Sometimes the EPD-N2 dosimeter does not respond above $30 \mu \mathrm{Sv}$ as shown in the plot. The results indicate that the EPD-N2 dosimeter has lower sensitivity than the DMC $2000 \mathrm{GN}$ and the reading is not reliable below a few hundred $\mu \mathrm{Sv}$.
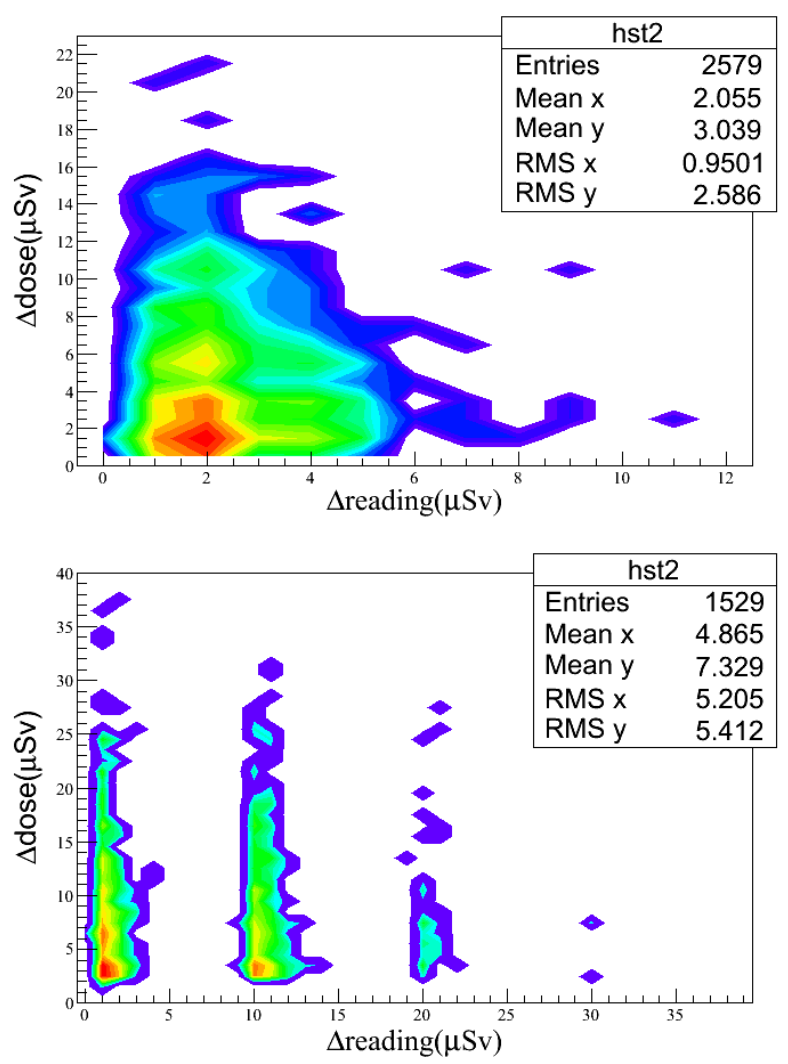

Figure 2. Contour plots of $\Delta$ reading and $\Delta$ dose to study the minimum dose to the dose indication for the DMC $2000 \mathrm{GN}$ (top) and for the EPD-N2 (bottom).

\subsection{Type B uncertainty evaluation from the statistical analysis}

For Type B uncertainty estimation the DMC 2000 GN and the EPD-N2 dosimeters were irradiated repeatedly. The average and the standard deviation of the calibration factor were evaluated from the statistical analysis and Type B uncertainty was calculated from the standard deviation. Irradiation time of each measurement was set to 30 minutes and the numbers of measurements were 33 for the DMC $2000 \mathrm{GN}$ and 30 for the EPD-N2. The reference doses were $764.4 \mu \mathrm{Sv}$ for the DMC $2000 \mathrm{GN}$ and $759.9 \mu \mathrm{Sv}$ for the EPD-N2. Uncertainties of the reference doses are $3 \%$ in $k=1$. From now on, coverage factors are in $k=1$ if they are not written. The averages of the readings were $(510.7 \pm 5.3)$ $\mu \mathrm{Sv}$ for the DMC $2000 \mathrm{GN}$ and $(517.0 \pm 10.6) \mu \mathrm{Sv}$ for the EPD-N2. Calibration factors were $1.47 \pm 0.02$ for the DMC $2000 \mathrm{GN}$ and $1.49 \pm 0.03$ for the EPD-N2 by dividing the dose by the reading. The results were compared to the calibration factors from the single measurements in section 3.1. The calibration factor 1.53 of the EPD-N2 in section 3.1 is in good agreement within two standard deviations. The result of the DMC $2000 \mathrm{GN}$ is better (within one standard deviation). The details are listed in the Table 1.

Type B uncertainty could be evaluated by assuming that the experimental data follow the normal distribution. Type B uncertainties were evaluated to be $6 \%$ $(=0.09 / 1.47)$ for the DMC $2000 \mathrm{GN}$ and $12 \%$ $(=0.18 / 1.49)$ for the EPD-N2. Type B uncertainty of the EPD-N2 is twice larger than that of the DMC 2000 GN. In this section, Type B uncertainties of the DMC 2000 GN and the EPD-N2 dosimeters were evaluated by a serial measurement of a single device. It is not certain that the dosimeter used for evaluation has typical one or not. Type B uncertainty evaluation with many dosimeters was done for EPD-N2 dosimeters and the result was in the next section.

Table 1. Data used for Type B uncertainty evaluations. The DMC 2000 GN and the EPD-N2 dosimeters of KRISS were tested.

\begin{tabular}{ccc}
\hline Target nucleus & EPD-N2 & DMC 2000 GN \\
\hline Measurement No. & 30 & 33 \\
Time $($ min. $)$ & 30 & 30 \\
Dose $(\mu \mathrm{Sv})$ & 757.9 & 764.4 \\
Reading $(\mu \mathrm{Sv})$ & $517.0 \pm 10.6$ & $510.7 \pm 5.3$ \\
Calibration factor & $1.49 \pm 0.03$ & $1.47 \pm 0.02$ \\
Standard dev. & 0.18 & 0.09 \\
Type B uncertainty & $12 \%$ & $6 \%$ \\
\hline
\end{tabular}

When a calibration factor of 1 count $=2.05 \mu \mathrm{Sv}$ for the DMC $2000 \mathrm{GN}$ and 1 count $=4.87 \mu \mathrm{Sv}$ for the EPD-N2 are assumed, the average counts could be evaluated from the Table 1 . They are $249(=510.7 \mu \mathrm{Sv}$ $/ 2.05 \mu \mathrm{Sv})$ for the DMC $2000 \mathrm{GN}$ and $106(=517.0 \mu \mathrm{Sv}$ $/ 4.87 \mu \mathrm{Sv}$ ) for the EPD-N2. The statistical variation of the DMC $2000 \mathrm{GN}($ the EPD-N2) is $6.3 \%(9.7 \%)$ which is nearly consistent with that obtained from the repeated 
measurement in Table 1. Although the count-to-dose conversion abovementioned is rather naïve, it probably accounts for the direct connection between "a minimum dose to the indication" and "the estimation of the Type B uncertainty".

\subsection{Type B uncertainty evaluation from the calibration factor distribution}

As mentioned in Experiment section, 153 EPD-N2 dosimeters were calibrated more than 5 times and the number of calibration factors was 1,208. EPD-N2s are in use at nuclear power plants in Korea (Uljin, Wolsong, Kori, and Youngkwang nucalr power plants). They were also calibrated with ${ }^{252} \mathrm{Cf}$ source at KRISS. Doses delivered to EPD-N2 calibrations were approximately 1 $\mathrm{mSv}$. Dosimeters are calibrated every 6 months which is determined by the nuclear power plants.

The number of calibration factors is large but it is hard to compare the calibration factors directly because the calibration factors of each device are not equal to each other. Because Type B uncertainty is a common characteristic to all EPD-N2 dosimeters, it could be possible to make normal distribution with the calibration factors: divide EPD-N2 calibration factors by their average to make the normalized normal distribution with the unit mean.

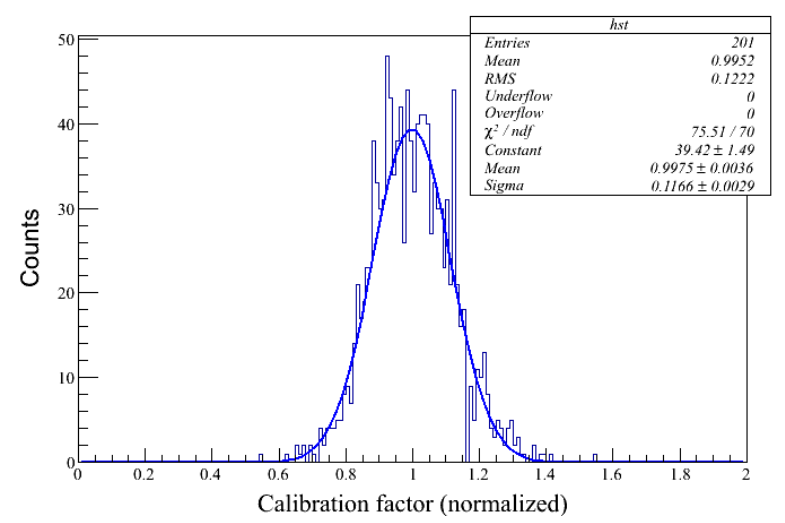

Figure 3. Normalized calibration factor distribution of EPD-N2 dosimeters.

All 1,208 calibration factors of 153 EPD-N2s were grouped to make the normalized calibration factor distribution as shown in Figure 3. The distribution was fitted with the Gaussian function. The mean of the Gaussian function was $0.9975 \pm 0.0036$ and its standard deviation was $0.1166 \pm 0.0029$. The standard deviation 0.1166 is in good agreement with the result of the serial measurement of a single EPD-N2. Type B uncertainty is $12 \%(=0.1166 / 0.9975)$ which is the same value in Table 1. Therefore, the uncertainty of Type B of EPD-N2 dosimeter was estimated to be $12 \%$.

\section{Conclusion}

In this study, dose dependence of calibration factors and minimum dose to the dose indication for DMC 2000 GN and EPD-N2 dosimeters were investigated with ${ }^{252} \mathrm{Cf}$ source neutrons in the neutron irradiation room at Korea Research Institute of Standards and Science. The neutron doses up to around $10 \mathrm{mSv}$ were irradiated. The calibration factor of EPD-N2 was stable above neutron dose around $2 \mathrm{mSv}$ and that of DMC $2000 \mathrm{GN}$ was stable above around $1 \mathrm{mSv}$.

Type B uncertainties of DMC $2000 \mathrm{GN}$ and EPD-N2 were evaluated to be $6 \%$ and $12 \%$ from the serial measurements. For EPD-N2, 1,208 calibration factors of 153 dosimeters were normalized to make the normalized normal distribution and Type $\mathrm{B}$ uncertainty was evaluated by fitting the distribution with Gaussian function. The result was in good agreement with the result from the serial measurement.

\section{Acknowledgements}

This work was supported by the Korea Research Institute of Standards and Science under the project "Development of measurement standards for medical radiation", grant 12011031 and the National Research Foundation of Korea (NRF) grant NRF-2010-002695 funded by the Korea government (MEST).

\section{References}

[1] ICRP Publication 74, Conversion coefficients for use in radiological protection against external radiation, Annals of the ICRP 27, 4 (1996).

[2] J. H. Kim, H. Park and K.-O. Choi, Calibration of neutron personal dosimeters in a realistically simulated neutron irradiation room, Radiat. Meas. 45 (2010), pp.1544-1547.

[3] H. Park, J. Kim and K.-O. Choi, Neutron calibration facility with radioactive neutron sources at KRISS, Radiat. Prot. Dosim. 126 (2007), pp.159-162. 